\title{
Hématome spontané du muscle psoas iliaque avec cruralgie déficitaire
}

\section{Spontaneous Iliopsoas Hematoma with Femoral Nerve Palsy}

\author{
M. Mihalcea-Danciu • L. Bejinariu $\cdot$ P. Bilbault
}

Reçu le 6 février 2015; accepté le 30 mars 2015

(C) SFMU et Lavoisier SAS 2015

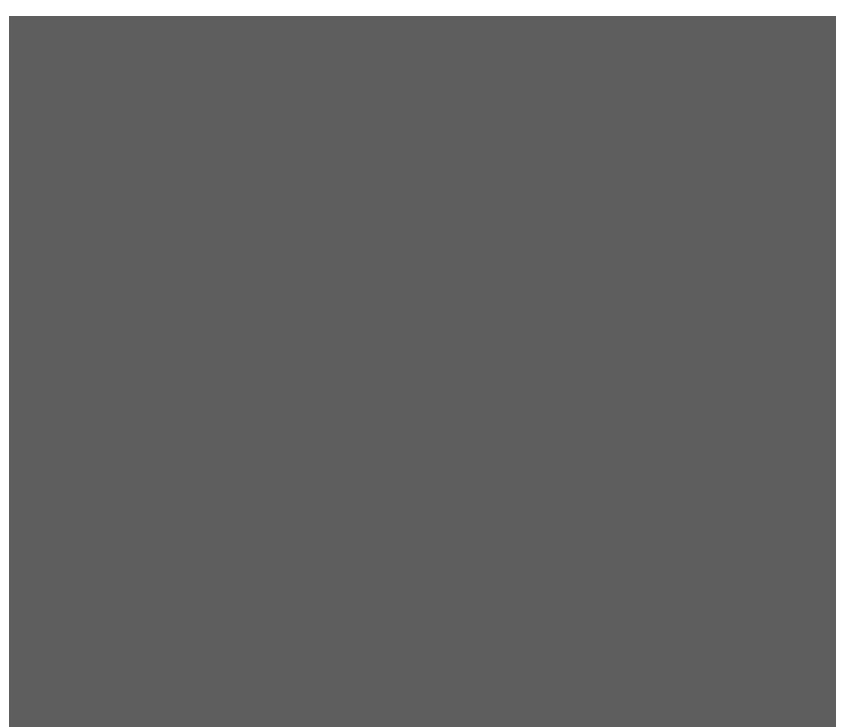

Une femme de 80 ans consulte aux urgences pour un déficit moteur isolé du membre inferieur droit installé depuis $24 \mathrm{~h}$ avec sensation d'engourdissement. Il n'y avait pas de notion de traumatisme récent. La patiente prenait du Fluindione suite à plusieurs embolies pulmonaires. L'examen neurologique retrouvait un déficit moteur isolé du quadriceps et des ischiojambiers droits coté $1 / 5$, associé à une atteinte de la sensibilité au niveau de la face antéro-interne de la cuisse droite. Le reste d'examen était normal. L'INR (International Normalized Ratio) était à 8,24. Le scanner abdominopelvien réalisé en urgence montrait un hématome du muscle iliaque droit, mesurant $60 \mathrm{~mm}$ d'épaisseur sans saignement actif décelé (Fig. 1). Cette pathologie est une entité rare dont l'incidence varie entre $0,6 \%$ et $6,6 \%$ [1]. Une cruralgie déficitaire chez un patient sous anticoagulants, même sans traumatisme, doit faire évoquer au clinicien l'hématome du muscle psoas-iliaque et conduire à la réalisation d'un scanner en urgence. Il n'existe pas de consensus concernant la prise en charge chirurgicale et/ou embolisation quand il existe un saignement actif. Notre patiente a bénéficié d'une intervention chirurgicale après l'antagonisation par concentré de complexes prothrombiniques et vitamine $\mathrm{K}$, selon les recommandations actuelles [2], avec une bonne évolution.

\section{Références}

1. Parmer SS, Carpenter JP, Fairman RM, et al (2006) Femoral neuropathy following retroperitoneal hemorrhage: case series and review of the literature. Ann Vasc Surg 20:536-40

2. Tazarourte K, Riou B, Tremey B, et al (2014) Guidelineconcordant administration of prothrombin complex concentrate and vitamin $\mathrm{K}$ is associated with decreased mortality in patients with severe bleeding under vitamin $\mathrm{K}$ antagonist treatment (EPAHK study). Crit Care 18:R81

M. Mihalcea-Danciu $(\bowtie) \cdot$ L. Bejinariu $\cdot$ P. Bilbault Service d'accueil des urgences, CHU de Strasbourg, hôpital de Hautepierre, 1 avenue Molière,

F-67098 Strasbourg, France

e-mail : mihalcea78@yahoo.com

P. Bilbault

EA 3072, FMTS, Faculté de médecine, université de Strasbourg,

4 rue Kirschleger, F-67000 Strasbourg 